\section{A combinatorial condition for planar graphs').}

By

\section{Sa unders Mac Lan e (Cambridge, Mass.).}

1. Introduction. Kurato $\mathrm{ski}{ }^{2}$ ) has proven that a topological graph is planar, i. e., that it can be mapped in a 1-1 continuous manner on the plane, if and only if it contains no subgraph having either of two specific forms. Whitine ${ }^{3}$ ) has shown that a graph is planar if and only if it has a combinatorial "dual". This paper establishes another combinatorial condition that a graph be planar. This condition may be stated in terms of ordinary combi. natorial concepts:

Theorem I. A combinatorial graph is planar if and only if the graph contains a complete set of circuits such that no arc appears in more than two of these circuits.

A graph in the plane divides the plane into a number of regions, and each region is bounded by one or more circuits. These boundary circuits, with certain omissions, can be readily shown to form a complete set, and obviously no arc can appear on more than two of these boundaries. Hence the necessity of our condition is immediate (cf. § 5). The sufficiency proof is largely combinatorial in character. It is advantageous to first reduce the problem to the case of the non-separable graphs (cf. § 3) considered by Whitney. By removing a suitable arc (or ares) from any non-separable graph we obtain a simpler non-separable graph. We first embed this simpler graph

1) Presented to the American Math. Society, April 11, 1936

2) C. Kuratowski, Fund. Math. Vol. XV (1930), pp. $271-283$. He considers a more general point set than a graph.

3) H. Whitney, Non-separable and planar graphs, 'Trans. Amer. Math. Soc. 34 (1932), pp. 339-362. We refer to this paper as Whitney I. in the plane, then show from the assumed condition that the remaining arc (or arcs) can be added in the plane $(\$ \$ 4,5)$.

By strictly combinatorial means the criterion of Theorem I will be shown equivalent (Theorem II, $\S 6$ ) to the existence of a dual. This is in turn known ${ }^{1}$ ) to be equivalent (combinatorially) to the condition of Kuratowski.

2. Definitions. A combinatorial graph $G$ consists of a finite set of elements $a, b, c, \ldots$ called "arcs", and a finite set of "vertices" $p, q, r, \ldots$, such that each are $b$ "joins" exactly two vertices $p$ and $q$. Then $p$ and $q$ are the ends of $b$ or are on $b$, while $b$ may be denoted by $p q$. We assume that each vertex is on at least one are ${ }^{2}$. Any set of ares in $G$, together with all the vertices on these ares, form themselves a subgraph of $G$. Each subgraph is determined by its arcs. If $m>1$ and if $p_{1}, p_{2}, \ldots, p_{m}$ denote distinct vertices, then a subgraph $C$ with ares $p_{1} p_{2}, p_{2} p_{3}, \ldots, p_{m-1} p_{m}, p_{m} p_{1}$ is a circuit, a subgraph $D$ with $\operatorname{arcs} p_{1} p_{2}, p_{2} p_{3}, \ldots, p_{m-1} p_{m}$ is a chain with ends $p_{1}$ and $p_{m}$, and the chain $D$ is suspended if $p_{1}$ and $p_{m}$ are the only vertices of $D$ on three or more $\operatorname{arcs}$ of $G$. If $A$ and $B$ are subgraphs, then $A \cap B$ is the subgraph containing those ares in both $A$ and $B, A+B$ contains those ares in either $A$ or $B$, and $G-A$ contains all arcs of $G$ not in $A$.

If a graph $G$ has $E(G)$ edges, $V(G)$ vertices, and $P(G)$ connected pieces, then

$$
R(G)=\nabla(G)-P(G), \quad N(G)=E(G)-V(G)+P(G)
$$

are respectively the rank and nullity of $G$. A sum modulo 2 of circuits $C_{1}+C_{2}+\ldots+C_{m}$ is the subgraph containing all ares present in an odd number of the $C_{i}$ 's. The circuits $C_{1}, C_{2}, \ldots, C_{n}$ form a complete set in $G$ if every circuit in $G$ can be expressed uniquely as a sum $\bmod 2$ of certain of the $C_{i}^{\prime}$ 's. Every $G$ contains at least one complete set of $n=N(G)$ circuits.

A planar topological graph $H$ consists of a finite number of ares $(1-1$ bi continuous images of line segments) in the plane intersecting, if at all, only at their endpoints. These arcs and their endpoints, considered as elements and possibly renamed, form a combinatorial graph $H^{\prime}$. Any such combinatorial graph $H^{\prime}$ is called planar, and $H$ is a map of $H^{\prime}$.

3. Non-separable graphs. A graph $G$ is separable if it has two subgraphs $F_{1}$ and $F_{2}$ such that $F_{1}+F_{2}=G$, while $F_{1}$ and $F_{2}$ have no ares and at most one vertex in common. If $F_{1}$ and $F_{2}$ have no common vertex, they are not connected; if they have one common vertex $p, p$ is called a cut vertex of $G$. In either event $G$ may be separated into $F_{1}$ and $F_{2} . F_{1}$ is either non-separable, or can itself be separated into $F_{3}$ and $F_{4}$, and likewise for $F_{2}$. Repetition of this finally yields subgraphs $G_{1}, G_{2}, \ldots, G_{m}$ which are no longer separable. These non-separable components of $G$ are always the same, no matter

1) H. Whitney, Planar Graphs, Fund. Math. XXI (1933), pp. 73-81 We refer to this paper as Whitney II.

2) This exclusion of "isolated" vertices obviously does not affect Theorem I. 
how the separation is carried out $\left.{ }^{\mathbf{1}}\right)$. On the other hand, tiwo arcs $\angle a$ and $b$ in $G$ are oyclically connected if $a=b$ or if there is a circuit in $G$ containing both $a$ and $b$. It can be proven that the relation " $a$ is cyclically connected to $b$ " is symmetric and transitive and that it holds if and only if ${ }^{2}$ ) $a$ and $b$ belong to the same non-separable component of $G$. This result gives an "invariant" definition of non-separable components, and so again proves their uniqueness.

Theorem 3.1. If $G$ is non-separable and has nulluty greater than 1 , and if $R$ is a circuit in $G$, then there is ${ }^{3}$ ) in $R$ a chain $A$ which is suspended in $G$ and whose removal leaves a non-separable graph $G-A$ of nullity $N(G)-1$.

The proof proceeds by building up $G$ from a sequence of nonseparable subgraphs $H_{1} \subset H_{2} \subset H_{3} \subset \ldots \subset G$. As $N(G)>1$, there is an are $a_{1}$ not in $R$. Pick a circuit containing $a_{1}$ and an are of $R$, and call this circuit $H_{1}$. If $H_{m-1} \neq G$ has been chosen, $H_{m}$ is constructed thus: First pick an are $a_{m}$ in $G-H_{m-1}$, such that $a_{m}$ is not in $R$ unless $G-H_{m-1}$ contains only ares of $R$. Since $G$ is non-separable, there is a circuit $D$ containing $a_{m}$ and an are of $H_{m-1}$. Denote by $A_{m}$ the piece of $D$ containing $a_{m}$ and extending in each direction from $a_{m}$ to the first vertex of $H_{m-1}$. Then choose $H_{m}$ as $H_{m}=H_{m-1}+A_{m}$.

Each subgraph $H_{m}$ is non-separable, as we now show by induction. The circuit $H_{1}$ must be non-separable. If $H_{m-1}$ is non-separable, then the arcs of $A_{m}$ are cyclically connected to the rest of $H_{m}$ by the circuit consisting of $A_{m}$ and a chain $B$ in $H_{m-1}$ joining the ends of $A_{m}$, so that $H_{m}$ is non-separable.

We next prove that $R \subset H_{m}$ implies $H_{m}=G$. By the construction of $H_{1}, R \subset H_{1}$ is impossible. Hence let $m>1$ be the smallest integer for which $R \subset H_{m}$. Then $R$ is not contained in $H_{m-1}$, and there is an are $b$ of $R$ not in $H_{m-1}$. Denote by $E$ the piece of $R$ which contains $b$ and extends along $R$ in each direction from $b$ up to the first vertex of $H_{m-1}$. This chain $E$ is in $R$, while $R \subset_{-} H_{m}$, so

1) Whitney I, Theorem 1.2 .

2) A similar proof in Whitney I, Theorem 7. Cf. also Kuratowski ot Whyburn, Sur les éléments cycliques et leurs applications, Fund. Math. XVI (1930), pp. 305-331.

a) This includes the special case of this Theorem, which was proven by Whitney (I, Theorem 18), and which does not require $A \subset R$. Theorem 3.1. can also be proven by an induction from Whitney's Theorem. that $E \subset H_{m}=H_{m-1}+A_{m}$. By construction, $E$ has no ares on $H_{m-1}$, so $E \subset A_{m}$. $A_{m}$ was chosen to have its ends and no other vertices in common with $H_{m-1}$. $E$ has the same property. Thus $E$ is a subchain of $A_{m}$ with the same ends as $A_{m}$, so that $E$ must equal $A_{m}$. Now if $G-H_{m-1}$ were not contained in $R, A_{m}$ would contain the arc $a_{m}$ not in $R$, so that $A_{m}$ cannot equal $E$, which is in $R$. Because of this contradiction, $G-H_{m-1}$ must be contained in $R$. But $H_{m}$ contains all of $H_{m-1}$ by construction and all of $R$ by assumption, and so contains all of $G$. Hence $R \subset H_{m}$ implies $H_{m}=G$ and $A_{m} \subset R$.

Each $A_{m}$ is a suspended chain in $H_{m}$, so that its addition to $H_{m-1}$ increases the number of arcs by 1 more than the number of vertices and so increases the nullity by 1 (cf. (1)). Thus $N\left(H_{m}\right)=m$. The construction process finally stops with an $H_{n}=G$, and this $n$ must be $N(G)$. The last added chain $A=A_{n}$ is a suspended chain in $H_{n}=G$ and is contained in $R$ (see the paragraph above), while $G-A$ has nullity $n-1$, just as required in the Theorem.

Separable graphs can also be built up in a standard fashion:

Theorem 3.2. If $G$ is separable, then there is a non-separable component $H$ of $G$ such that $H$ and $G-H$ have at most one vertex in common.

Proof: Pick out any component $H_{1}$ of $G$. If $H_{1}$ does not have the desired property, there is a component $H_{2}$ in $G-H_{1}$ with a vertex $p_{1}$ in common with $H_{1}$. If $H_{2}$ does not have the desired property, there is a component $H_{3} \neq H_{2}$ containing a vertex $p_{2} \neq p_{1}$ in common with $H_{2}$. Were $H_{3}=H_{1}$, then chains of $H_{2}$ and $H_{1}$ joining $p_{1}$ to $p_{2}$ would form a circuit containad in no one component, an impossibility. Hence $H_{1}, H_{2}$ and $H_{3}$ are distinct. If $H_{3}$ does not have the desired property, we find a new component $H_{4}$, etc. The graph is finite, so the process must end with a component $H_{m}$ with but one vertex in common with $G-H_{m}$, as required.

4. The Induction Process. Consider a combinatorial graph $G$ which satisfies the condition of Theorem $I$. That is, assume that $G$ contains a complete set of circuits

$$
C_{1}, C_{2}, \ldots, C_{n}
$$

which contain no arc more than twice. We call such a set (2) a 2-fold complete set. The $C_{i}$ are independent, so that the sum

$$
R=C_{1}+C_{2}+\ldots+C_{n}
$$

is not zero. We call $R$ the rim of $G$. It has the following property: 
Lemma 4.1. For a non-separable $G$, the rim (3) is a rireuit.

Suppose instead that $R$ is not a circuit. It is then a cycle (i. e., each vertex is on an even number of ares of $l R$ ) and so contains a proper subcircuit $D$. The representation of $D$ in terms of the complete set (2) may be written, if the $G_{i}$ 's are suitably renumbered, as

$$
D=C_{1}+C_{2}+\ldots+C_{m}
$$

As $D \neq R, n$ exceeds $m$. Hence neither of the graphs

$$
F_{1}=O_{1}+O_{2}+\ldots+O_{m}, \quad F_{2}=O_{m+1}+O_{m+2}+\ldots+O_{n}
$$

is void. We shall show that $G$ separates into $F_{1}^{\prime}$ and $H_{2}$. An are $b$ of $F_{1} \cap F_{2}$ must be in one of the first $m$ ('s and in one of the last $(n-m) C$ 's. Since (2) is a 2 -fold set, $b$ is in no more of the $\theta$ 's. Thus $b$ is in just one summand of $D$ and in two summands of $R$, $\bmod 2$, so that $b$ is in $D$ and not in $R$, although $D \subset R$. This contradiction shows that $F_{1}$ and $F_{2}$ have no ares in common.

As $G$ is non-separable, there is a circuit $W$ containing an are of $F_{1}$ and one of $F_{2}$. The representation of $E$ in the complete set is

$$
E=\Sigma^{\prime \prime} C_{t}+\Sigma "{ }^{\prime \prime} C_{l}
$$

where the first sum runs over some of the indices from 1 to $m$ and the second sum over some of the remaining indices. Since $E$ contains edges of both $F_{1}$ and $F_{2}$, neither sum is void. The first sum $E^{\prime}=\Sigma^{\prime} C_{i}(\bmod 2)$ is thus not equal to $K$. But $E^{\prime}$ is contained in $F_{1}^{\prime}$, so that none of its ares can be contained in the circuits $\theta_{m+1}, \ldots, O_{n}$ of $F_{2}$. Therefore $E^{\prime} \subset E$. The circuit $E$ has a proper subcycle $E^{\prime}$, an impossibility. Hence $R$ is necessarily a circuit.

In a non-separable planar graph there must be a region boundary $C_{i}$ which abuts on the outside boundary $R$ only along a single chain. The corresponding combinatorial result can be stated thus:

Lemma 4.2. If $G$ is non-separable and has nullity greater than 1, then there is a suspended chain $A$ in $G$ such that

(i) $G-A$ is non-separable of nullity $N(G)-1$;

(ii) $A$ is contained in $R$ and in one and only one $O_{l}$, say in $G_{n}$,

(iii) $C_{1}, C_{2}, \ldots, C_{n-1}$ form a 2-fold complete set for $G-A$,

(iv) The ends $p$ and $q$ of $A$ are on $R^{\prime}$, where

$$
R^{\prime}=O_{1}+C_{2}+\ldots+O_{n-1}
$$

(v) $R^{\prime}$ consists of two chains $R-A$ and $C_{n}-A$ joining $p$ to $q$.
Pr o of: Pick a suspended chain $A \subset R$ as in Theorem 3.1. Then $G-A$ is non-separable, as in (i). Since $G$ is non-separable, each are of $A$, and hence all of $A$, is contained in some circuit. Any circuit is a sum, mod 2, of the $C^{\prime}$ 's, so that $A$ is contained in some $C_{i}$. By renumbering we can make $A \subset C_{n}$. Since $A$ is in $R$ and at most two $C$ 's, $A$ can be in no other $C_{i} \neq C_{n}$, as asserted in (ii). The circuits $C_{1}, C_{2}, \ldots, C_{n-1}$ are thus in $G-A$, and they are independent in $G-A$ as in $G$. Since there are at most $n-1=N(G-A)$ independent circuits in $G-A$, these $n-1$ circuits form a 2 -fold complete set in $G-A$, as stated in (iii).

We next show $A=C_{n} \cap R$. For suppose $b$ were an are in $C_{n} \cap R$ but not in $A$. Then $b$ is in $G-A$, hence is in a circuit $D$ of $G-A . D$ is representable, by (iii), as a sum mod 2 of some of $C_{1}, C_{2}, \ldots, C_{n-1}$. Yet $b$ is in $C_{n}$ and $R$, hence can be in no other $C_{l}$, and so is not in the representation of $D$, contrary to $b \in D$. No such edge $b$ is possible, so that $A=C_{n} \cap R$.

By (4), $R^{\prime}=R+C_{n}(\bmod 2)$, so that $R^{\prime}$ consists of all arcs in $R$ or in $C_{n}$ but not in both. Those in both are in $R \cap C_{n}=A$, so that $R^{\prime}$ must consist of the arcs of $R-A$ and of $C_{n}-A$. As $R$ is a circuit, $R-A$ (and also $C_{n}-A$ ) is a chain joining the ends $p$ and $q$ of $A$. $R-A$ is in $R^{\prime}$, so that its ends $p$ and $q$ are in $R^{\prime}$. This gives the last conclusions (iv) and (v) of the Lemma.

\section{The Sufficiency Proof.}

Theorem 5.1. A non-separable graph $G$ with a 2-fold complete set (2) can be mapped on the plane in such a way that each $C_{i}$ becomes the boundary of one of the finite regions into which $G$ divides the plane, while $R$ becomes the boundary of the exterior region.

The proof will be by induction on the nullity $N(G)$. To avoid irrelevant topology, we shall show more explicitly that $G$ can be embedded in such a way that each are of $G$ becomes a broken line segment, while $R$ becomes an equilateral triangle. In the first case, if $N(G)=1, G$ is non-separable and so is simply a circuit (ef. Whitney I, Theorem 10); hence it can be mapped on the plane. If $N(G)>1$, then Lemma 4.2 yields a suspended chain $A$ such that $G-A$ is non-separable, has a 2 -fold complete set, and has a smaller nullity. By the induction assumption, $G-A$ can be mapped on the interior and boundary of a triangle, with $R^{\prime}$ on the boundary. By Lemma 4.2, (iv), the ends $p$ and $q$ of $A$ are already on the boundary $R^{\prime}$, and 
by (v) the remainder $C_{n}-A$ of $C_{n}$ is one of the aress of this boundary joining $p$ to $q$. Hence we can add a new broken line segment $A^{\text {* }}$ outside the triangle and with ends $p$ and $q$ in such a way that $A^{*}$ and $C_{n}-A$ together form the boundary of the new finite region. Then by ( $v$ ) the boundary of the new exterior region is $R=A^{*}+$ $+(R-A)$. The induction proof will be complete if we show that $R$ can be made an equilateral triangle.

To do this, let $G-A$ be mapped on the triangle with vertices $r, s$, and $t$ in the figure. Consider first the case when the are

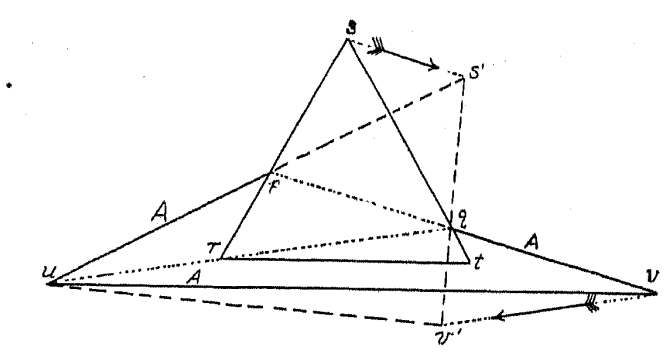

$C_{n}-A$ on the edge of this triangle contains two vertices $r$ and $t$ of the triangle. Map $A$ as the broken line $p u v q$ in the figure. To make the rim spuvqs a triangle, first shear ${ }^{1}$ ) the half plane which contains $s$ and has the edge $p q$ until the new position $p s^{\prime}$ of $p s$ is a prolongation of $u p$. Then shear the half plane with the edge $u q$ until $s^{\prime} q v$ is a straight line $s^{\prime} q v^{\prime}$. The new rim is now a triangle $u s^{\prime} v^{\prime}$. It may be made isosceles by a shear with edge $v^{\prime} s^{\prime}$, then equilateral by a compression toward this edge. As the shears used carry straight lines into broken lines, we do obtain a broken-line map of $G$ on an equilateral triangle. The other cases, when $O_{n}-A$ contains other vertices $r, s$, or $t$ of the map $G-A$, may be similarly treated.

Theorem 5.2. A graph $G$ with a 2-fold complete set of circuits can be mapped on the plane in such a way that each are becomes a broken line-segment.

If $G$ is separable, it can be reduced to non-separable components $H_{1}, H_{2}, \ldots, H_{m}$. Each component $H_{i}$ which is not a single

1) A shear in a half-plane moves each point $P$ parallel to the edge a dism tance which is a constant times the distance of $P$ from that edge. are contains one or more of the given complete set of circuits (2), say the circuits $C_{1}, C_{2}, \ldots, C_{k}$. These circuits form a complete set for $H_{i}$ : In the first place, they are independent $(\bmod 2)$; secondly, any circuit $D$ in $H_{i}$ is expressible in terms of all the $C_{\text {'s }}$ in the form $D=\Sigma^{\prime} C_{j}+\Sigma^{\prime \prime} C_{j}(\bmod 2)$, where the first sum runs over certain indices $j \leqq k$, the second sum over certain indices $j>k$. Then $D-\Sigma^{\prime} C_{j}$ has ares only in $H_{i}$ and is equal to $\Sigma^{\prime \prime} C_{j}$, which has arcs only in other components, and so must be void. $D=\Sigma^{\prime} C_{j}$ $(\bmod 2)$ is thus represented in terms of $C_{1}, C_{2}, \ldots, C_{k}$, and these circuits are a 2 -fold complete set for $H_{i}$. Therefore $H_{i}$ is planar, by Theorem 5.1. Furthermore it will suffice to prove the Theorem for a connected graph $G$.

The proof proceeds by induction on the number of components. Choose one component, say $H_{m}$, so that $H_{m}$ and $G-H_{m}$ have but one vertex $p$ in common (Theorem 3.2), and make the induction assumption that $G-H_{m}$, with components $H_{1}, H_{2}, \ldots, H_{m-1}$, has already been mapped on the plane as a graph $F$. If $H_{m}$ has nullity zero, it is but a single arc and can be readily added to $F$ in the plane. If $N\left(H_{m}\right)>0$, then the vertex $p$ appears in at least one of the, circuits $C_{j}$ of the remaining component $H_{m}$. If $p$ is not already on the rim of $H_{m}$, we can make this the case by using a new 2 -fold complete set for $H_{m}$ like the old one except that one $C_{j}$ containing $p$ is replaced by the rim of $H_{m}$. Then $p$ is on the new rim $C_{j}$. By Theorem 5.1, map $H_{m}$ on the plane as the graph $H_{m}^{*}$, so that $p$ becomes a point $p_{1}^{*}$ on the outside boundary. But $p$ also appears in the map of $G-H_{m}$ as point $p_{2}^{*}$; it remains to fit these maps together so that $p_{1}^{*}$ and $p_{2}^{*}$ will coalesce.

To do this, cut the plane of $H_{m}^{*}$ into two halfplanes by a line through $p_{1}^{*}$ and shear each half plane until the boundary of $H_{m}^{*}$ makes at $p_{1}^{*}$ an angle $\alpha$ smaller than the angle $\beta$ between two of the adjacent (straight line) arcs which meet in the corresponding vertex $p_{2}^{*}$ of the map of $G-H_{m}$. Then shrink $H_{m}^{*}$ until it can fit inside the region which contains the angle $\beta$, place $H_{m}^{*}$ in this region and let the points $p_{1}^{*}$ and $p_{2}^{*}$ coalesce, thus forming the desired cut vertex $p^{*}$ in a planar map $\left.{ }^{1}\right)$ of $G=\left(G-H_{m}\right)+H_{m}$. All the transformations involved map the arcs of $G$ into straight or broken line segments.

1) Another proof, using more topology, is indicated in Whitney I, Theorem 27. 
This establishes the sufficiency of the eriterion of Theorem $\mathrm{I}$. It remains to verify its necessity. If each component of $G$ has a 2 -fold complete set of circuits, all these circuits together form a 2 -fold complete set for $G$; hence it suffices to consider the non-separable case.

Theorem 5.3. If $G$ is a non-separable planar graph with $N(G): 0$, then each finite region into which $G$ divides the plane has as boundary a circuit of $G$, and these circuits together form a 2-fold complete set, while their sum, mod 2, is the boundary of the external region.

For $N(G)=1$ this is the Jordan curve Theorem: in general, it follows by a simple induction on $N(G)$, using Theorem 3.1 and the fact that a cross-cut in the interior (exterior) of a Jordan curve cuts the interior (exterior) into two regions with suitable boundaries. This insures that each added are is on the boundary of at mosti tiwo regions.

6. A Combinatorial Construction of Duals. Whitnoy's condition for a planar graph, the existence of a combinatorial dual, must be equivalent to the existence of a 2 -fold complete set of circuits. We shall establish this equivalence by combinatorial arguments, thus giving another proof for the criteria of Whitney and Kuratowski for planar graphs.

A graph $G^{\prime}$ is a dual of a graph $G$ if there is a 1-1. correspondence between the arcs of $G$ and those of $G^{\prime}$ such that, if $H$ is any subgraph of $G$ and $H^{\prime}$ the subgraph containing the corresponding ares of $G^{\prime}$, then $R\left(G^{\prime}-H^{\prime}\right)=R\left(G^{\prime}\right)-N(H)$; (cf. (1)). A set $S^{\prime}$ of arcs in $G$ is a out set if $G-S$ has either more connected pieces or fewer vertices ${ }^{1}$ ) than $G$, and if neither of these results would hold were $S$ replaced by a proper subset of $S$ (Whitney II, p. 76). A graph has a dual if and only if each of its components has a dual (Whitney $\mathrm{I}$, Theorems 23 and 25), and has a 2 -fold complete set of circuits if and only if its components have such sets, by the arguments of the preceding section. Hence we restrict our attention to the nonseparable case.

Theorem II. A non-separable graph $G$ has a combinatorial dual if and only if it has a 2-fold complete set of oirouits.

1) Practically, this s cond case means that $G-S$ is discomnected and has one piece which is an isolat d vertex. We have excluded such vertices.
The trivial case when $G$ is a single arc will be omitted. First, let $G$ have the 2 -fold complete set (2), with $n>0$, and denote the $\operatorname{rim} R$ in (3) by $C_{n+1}$. Since every arc is on at least one circuit, and on at most two circuits of the complete set, each are is on exactly two of $C_{1}, \ldots, C_{n+1}$. Construct a new graph $G^{\prime}$ with vertices $p_{1}, \ldots, p_{n+1}$ corresponding to the circuits $O_{i}$ and with ares $b_{1}^{\prime}, b_{2}^{\prime}, \ldots, b_{t}^{\prime}$ in $1-1$ correspondence with the ares of $G$, such that an are $b_{j}^{\prime}$ has the ends $p_{i}$ and $p_{k}$ if the corresponding arc $b_{j}$ in $G$ is on the circuits $C_{i}$ and $C_{k}$. We shall prove that this "circuit graph" $G$ ' is in fact a dual of $G$, by means of a combinatorial analog of the Jordan curve Theorem:

Lemma 6.1. If $D$ is a circuit in $G$, then the corresponding subgraph $D^{\prime}$ in $G^{\prime}$ is a cut set in the circuit graph $G^{\prime}$.

Proof: $D$ has a representation in terms of the complete set of $C$ 's. Renumber the $C$ 's so that the first $k$ appear in this representation, and then use the definition (3) of $C_{n+1}$ to obtain

$$
D=C_{1}+C_{2}+\ldots+C_{k}=C_{k+1}+C_{k+2}+\ldots+C_{n+1} \quad(\bmod 2) .
$$

There is a corresponding subdivision of the vertices of $G^{\prime}$ into two sets $p_{1}, \ldots, p_{k}$ and $p_{k+1}, \ldots, p_{n+1}$. By the representation (5), $D^{\prime}$ consists of all the ares of $G^{\prime}$ which have one end in the first set of vertices and the other end in the second. Thus in $G^{\prime}-D^{\prime}$ no vertex of the first set is connected to any vertex of the second. However, the vertices of the first set are all connected to each other in $G^{\prime}-D^{\prime}$. For were some of the vertices, say $p_{1}, p_{2}, \ldots, p_{j}$, connected by no arc of $G^{\prime}-D^{\prime}$ to the remaining vertices, then the sum $E=C_{1}+$ $+\ldots+C_{j}(\bmod 2)$ of the corresponding circuits of $G$ would contain no arc also contained in $C_{j+1}, \ldots, C_{k}$ and so would contain only arcs in $D$. Thus $E$ would be a proper subcycle of the circuit $D$, a contradiction. Similarly the vertices of the second set are all connected to each other in $G^{\prime}-D^{\prime}$. The addition of any single are of $D^{\prime}$ connects the first set of vertices to the second, so that $D^{\prime}$ is a cut set, as asserted in the Lemma.

To show $G^{\prime}$ a dual of $G$ it suffices ${ }^{1}$ ) (Whitney II, Theorem 2) to show that a circuit in $G$ always corresponds to a cut sft of arcs in $G^{\prime}$, and conversely. The first half is the above Lemma. For the

1) At this point we could alternatively parallel the argument of Whitney I, Theorem 29, that a planar graph has a dual, replacing the Jordan curve Theorem by the above Lemma. 
converse, let $S^{\prime}$ be a cut set in $G^{\prime}$. Since by the proof of the Lumma. the cut set $D^{\prime}$ cuts $G^{\prime}$ into just two pieces, $G^{\prime}$ itself must be connected. Therefore $S^{\prime}$ cuts $G^{\prime}$ into two ,pieces". Let the first contain the (re-numbered) vertices $p_{1}, \ldots, p_{l}$; the second, the vertices $p_{k+1}, \ldots, p_{n+1}$. Then the corresponding circuits in $G$ give a cycle

$$
D=C_{1}+C_{2}+\ldots+C_{k}=C_{k+1}+C_{k+2}+\ldots+C_{n+1} \quad(\bmod 2)
$$

Every arc on $D$ belongs to one of the first $k C$ 's and to one of the remaining $C$ 's, and so corresponds to an are in $G^{\prime}$ connecting the first set of vertices to the second set. This arc must belong to the cut set, so that $D C S$. But the cycle $D$ must contain a subset $D_{1}$ which is a circuit. The corresponding set $D_{1}^{\prime}$ is, by Lemma 6.1, a cut set in $G^{\prime}$, although $D_{1}^{\prime} \subset S^{\prime}$. By definition a cut set has no proper subset which is a cut set, so that $S$ must be identical with the circuit $D_{1}$, and cut sets do correspond to circuits. This shows $q^{\prime}$ to be a dual of $G$.

Conversely, let $G$ have a dual $G$. To find a 2-fold complete set of circuits, note first that the non-separability of $G$ implies ${ }^{1}$ ) that of $G^{\prime}$. By the definition of a dual, the nullity $n$ of $G$ is the same as the rank $V\left(G^{\prime}\right)-1$ of $G^{\prime}$ (cf. (1)), so that $G^{\prime}$ has $n+1$ vertices $p_{1}, p_{2}, \ldots, p_{n+1}$. The set $D_{i}^{\prime}$ of all ares on any one vertex $p_{i}$ is a cut set in $G^{\prime}$, for its removal deletes $p_{i}$. Consequently the corresponding set of $\operatorname{arcs} D_{i}$ in $G$ must be a circuit, by the Theorem of Whitney quoted previously. These circuits $D_{1}, D_{2}, \ldots, D_{n+1}$ contain each are of $G$ exactly twice, so that their sum is zero $(\bmod 2)$. No other relation mod 2 is possible. For suppose instead that

$$
D_{1}+D_{2}+\ldots+D_{m}=0 \quad(\bmod 2) ; \quad m<n+1 .
$$

Then any arc of $G$ on one of these $D$ 's is on another one, so that any arc of $G^{\prime}$ with a first end on one of $p_{1}, \ldots, p_{m}$ has a second end on another one of these vertices, and $p_{1}, \ldots, p_{m}$ are not connected to the remainder of $G^{\prime}$. This contradicts the non-separability of $G^{\prime}$. Thus $D_{1}, \ldots, D_{n}$ are independent $(\bmod 2)$, are $n=N(G)$ in number, and so form a 2 -fold complete set of circuits. Theorem II is established. tial here.

1) Whitney I, Theorem 26. The exclusion of isolated vartices is essen-

\section{Freie Überdeckungen und freie Abbildungen.}

Von

\author{
H einz Hopf (Zürich).
}

\section{Einleitung.}

1. Den Ausgangspunkt für unsere Betrachtungen bildet die folgende Eigenschaft der $n$-dimensionalen Sphären, die zuerst von L. Lusternik und L. Schnirelmann, und dann noch einmal von $\mathrm{K}$. Borsuk entdeckt und bewiesen worden ist ${ }^{1}$ ):

Satz $\boldsymbol{A}_{n}$. Ist die n-dimensionale Sphäre $S^{n}$ mit $n+1$ abgeschlossenen Mengen überdeckt, so enthält wenigstens eine dieser Mengen ein antipodisches Punktepaar der Sphäre.

Die isolierte Stellung dieses interessanten Satzes reizt zu dem Versuch, ihn in ein System allgemeinerer Überdecküngssätze einzuordnen. Beginnt man bei einem solchen Versuch mit der Analyse des Falles $n=1$, so sieht man sofort, daß der Satz $A_{1}$ nur ein Korollar des folgenden viel allgemeineren Satzes ist:

Satz $\boldsymbol{A}_{1}^{*}$. Bilden die abgeschlossenen Mengen $F_{1}$ und $F_{2}$ eine Überdeckung des zusammenhängenden topologischen Raumes ${ }^{2}$ ) $R$, und ist $f$ irgend eine stetige Abbildung von $R$ in sich, so enthält wenigstens eine der beiden Mengen ein Punktepaar $\{x, f(x)\}$.

Denn da $R$ zusammenhängend ist, gibt es einen Punkt $x \in F_{1} \cdot F_{2}$, und das Püktepaar $\{x, f(x)\}$ gehört der Menge $F_{i}$ an, wenn diese den Punkt $f(x)$ enthält.

$\left.{ }^{1}\right)$ L. Lusternik et L. Schnirelmann, Méthodes topologiques dans les problèmes variationnels, Moskau 1930 (in russischer Sprache), S. 26, Lemma 1. K. Borsuk, Drei Sätze über die n-dimensionale Sphäre, Fund. Math. XX (1933), S. 177. Man vergl, auch P. Alexandroff und H. Hopf, Topologie I (Berlin 1936), S. 486-487. Dieses Buch wird im folgenden als A.-H. zitiert.

2) Unter einem topologischen Raum soll immer ein Raum verstanden werden, der die K uratowskischen Axiome erfüllt; s. A.-H. (cf. Fußnote ${ }^{1}$ )), S. 37 ff. Fundamenta Mathematicae. T. XXVIII. 